\title{
Dark current reduction in ultraviolet metal-semiconductor-metal photodetectors based on wide band-gap semiconductors
}

\author{
Serkan Bütün ${ }^{1}$, Mutlu Gökkavas ${ }^{2}$, HongBo Yu ${ }^{2}$, Vlodek Strupinski ${ }^{3}$ and Ekmel Özbay ${ }^{4}$ \\ ${ }^{1}$ Nanoteknoloji Araştıma Merkezi and Fizik Bölümü, Bilkent Üniversitesi, Ankara, Türkiye \\ ${ }^{2}$ Nanoteknoloji Araştıma Merkezi, Bilkent Üniversitesi, Ankara, Türkiye \\ ${ }^{3}$ Institute of Electronic Materials Technology, 01-919 Warsaw, Wolczynska 133 Str., Poland \\ ${ }^{+}$Nanoteknoloji Araştıma Merkezi and Fizik Bölümü and Elektrik ve Elektronik Mühendisliği Bölümü, \\ Bilkent Üniversitesi, Ankara, Türkiye
}

\begin{abstract}
Photodetectors on semi-insulating GaN templates were demonstrated. They exhibit lower dark current compared to photodetectors fabricated on regular GaN templates. Similar behavior observed in photodetectors fabricated on epitaxially thick SiC templates.
\end{abstract}

\section{Summary}

Here we present our work on metal-semiconductor-metal (MSM) photodetectors on semi-insulating GaN templates (Figure 1a). Epitaxial growth of the samples was performed in a metal organic chemical vapor deposition reactor. We also fabricate MSM photodetectors on regular unintentionally doped GaN template (Figure 1b) for an evenhanded comparison. Devices on semi-insulating template exhibited four orders of magnitude lower compared with devices on regular template (Figure 2). A dark current density of $1.96 \times 10^{-10} \mathrm{~A} / \mathrm{cm}^{2}$ at $50 \mathrm{~V}$ bias was measured for photodetectors on semi-insulating template. Spectral responsivity measurements were carried out. Photodetectors fabricated on the semi-insulating template was able to detect incident optical power of as low as $3 \mathrm{pW}$ at $360 \mathrm{~nm}$ illumination. 
(a)

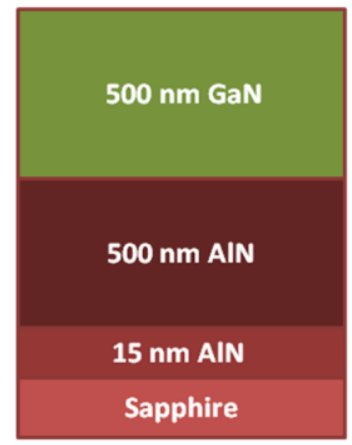

Semi-insulating GaN (b)

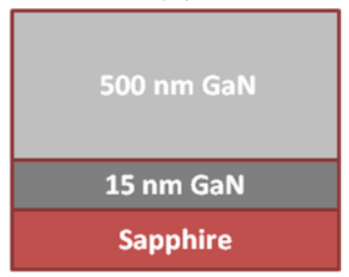

Unintentionally Doped GaN
Figure 1: Epitaxial structures of (a) semi-insulating (b) unintentionally doped GaN templates.

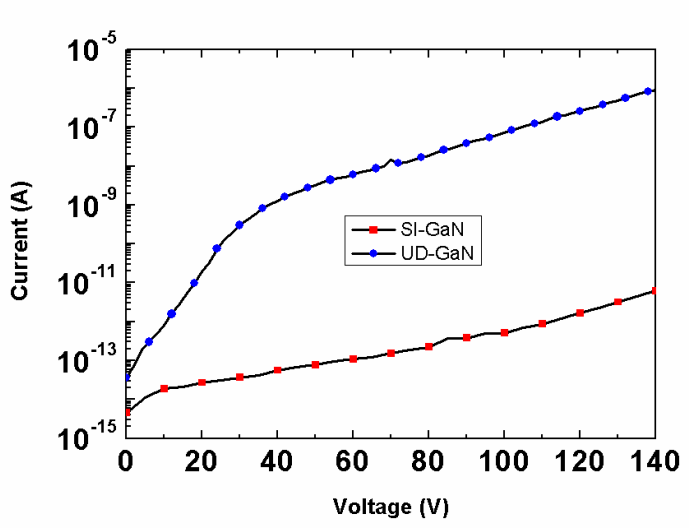

Figure 2: Current-Voltage characteristics comparison of the MSM photodetectors.

We also show our efforts on SiC based metal-semiconductor-metal photodetectors epitaxially grown on $\mathrm{SiC}$ templates. The epitaxial growth was performed by using a chemical vapor deposition system. The effect of the growth thickness on device performance was investigated by undoped SiC layers of different thicknesses (5 micron and 10 micron), which were grown on $4 \mathrm{H}-\mathrm{SiC}$ substrate. The photoluminescence intensity of the 10 micron thick template was twice as high (Figure 3 ). The devices grown on the 10 micron thick template showed a dark current density $4.4 \times 10^{-11} \mathrm{~A} / \mathrm{cm}^{2}$ under $50 \mathrm{~V}$ reverse bias voltage, which was four orders of magnitude lower than those devices grown on the 5micron template (Figure 4). The peak responsivity for the 10 micron thick sample was $7.2 \times 10^{-2} \mathrm{~A} / \mathrm{W}$, under $303 \mathrm{~nm}$ UV illumination and $25 \mathrm{~V}$ reverse bias. The corresponding peak responsivity for the 5 micron thick sample was $7.3 \times 10^{-2} \mathrm{~A} / \mathrm{W}$, at $273 \mathrm{~nm}$ UV illumination and $25 \mathrm{~V}$ reserve bias.

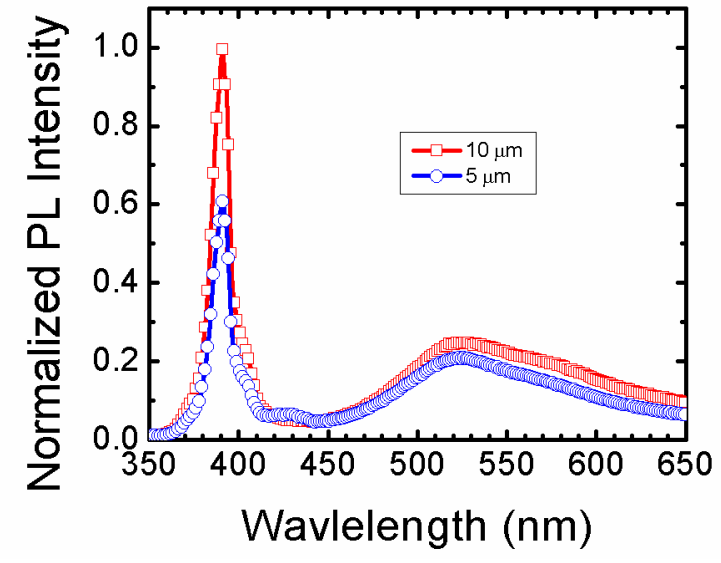

Figure 3: Normalized PL spectrum of the SiC epitaxial layers. Data is normalized to maximum of the red curve.

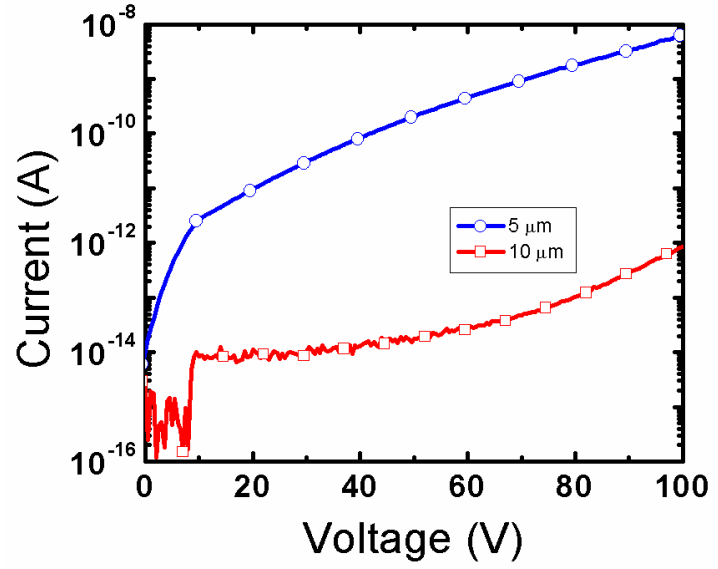

Figure 4: IV Characteristics of the SiC MSM photodetectors. 